\title{
RELATIVE GROTHENDIECK RINGS'
}

\author{
BY T. Y. LAM AND I. REINER
}

Communicated by I. Reiner, January 17, 1969

1. Introduction. Let $H$ be a subgroup of the finite group $G$, and let $\Omega$ be a field of characteristic $p$, where we assume $p \neq 0$ to avoid trivial cases. Form the free abelian group $a$ on the symbols $[M]$, where $M$ ranges over the representatives of a full set of isomorphism classes of finitely generated left $\Omega G$-modules (hereafter called " $G$-modules" for brevity). Let $\&$ be the subgroup of $a$ generated by all expressions $[M]-[L]-[N]$, where

$$
0 \rightarrow L \rightarrow M \rightarrow N \rightarrow 0
$$

ranges over all $H$-split exact sequences of $G$-modules. The relative Grothendieck ring $a(G, H)$ is defined as $Q / \otimes$, acquiring a ring structure by letting $[M]\left[M^{\prime}\right]=\left[M \otimes_{\Omega} M^{\prime}\right]$ where $G$ acts diagonally on the tensor product.

The structure of $a(G, H)$ has been investigated by the authors in two earlier articles [1], [2]. In the extreme case where $H=1$, the ring $a(G, 1)$ is just the ring of generalized Brauer characters of $G$. On the other hand, $a(G, G)$ is the representation ring of $G$, gotten by considering $G$-modules relative to direct sum. In general, $a(G, K)$ $\cong a(G, H)$ if $H$ is a Sylow p-subgroup of $K$, and so there is no loss of generality in assuming hereafter that $H$ is a $p$-subgroup of $G$.

Let $k(G, H)$ be the ideal of $a(G, G)$ spanned by all $(G, H)$-projective $G$-modules. The Cartan map

$$
\kappa: k(G, H) \rightarrow a(G, H)
$$

is defined by $[M] \rightarrow[M]$, and as shown in $[2], \kappa$ is a monomorphism. Furthermore, the cokernel of $\kappa$ is a $p$-torsion abelian group when $H \Delta G$.

We have previously established

Theorem 1 [1, Theorems 3.4 and 4.4]. If $H \Delta G$, where $H$ is a cyclic p-group, then a $(G, H)$ has a finite free $Z$-basis. Furthermore, if $G$ is a semidirect product $H \cdot A$, then there is a $Z$-isomorphism

$$
a(G, H) \cong a(H, H) \otimes_{z} a(G, 1) .
$$

This isomorphism is in fact a ring isomorphism when $G$ is the direct product $H \times A$.

\footnotetext{
I This research was partially supported by the National Science Foundation.
} 
TheOREM 2 [2, Theorem 3.2]. Let $G$ be a semidirect product $P \cdot K$, where $P$ is a normal p-subgroup of $G$, and let $H$ be any subgroup of $K$. Then the restriction map $a(G, H) \rightarrow a(K, H)$ is a ring isomorphism.

Theorem 3 [2, Theorem 5.1]. Let $G$ be a direct product $B \times K$, and let $H$ be any subgroup of $K$. Assume that $\Omega$ is a splitting field for $G$ and all of its subgroups. Then there is a ring isomorphism

$$
a(G, H) \cong a(B, 1) \otimes_{z} a(K, H) .
$$

2. New results. Making use of the above theorems, we have recently obtained several interesting generalizations, as follows.

TheOREM 4. Let $H$ be a cyclic subgroup of a p-group $G$. Then the restriction map $a(G, H) \rightarrow a(H, H)$ is a ring isomorphism. The cokernel of the Cartan map $\alpha$ is a finite p-group.

THEOREM 5. Let $H$ be a normal p-subgroup of an aribtrary finite group $G$, and assume that there exists a set of coset representatives of $H$ in $G$ each of which centralizes $H$. Then there is a ring isomorphism

$$
a(G, H) \cong a(H, H) \otimes_{z} a(G, 1),
$$

and hence $a(G, H)$ is $Z$-free.

The proof of Theorem 5 is based on the following special case, interesting in its own right.

THEOREM 6. Let $H$ be a normal subgroup of the p-group $G$, and suppose there exists a set of centralizing coset representatives of $H$ in $G$. Then the restriction map $a(G, H) \rightarrow a(H, H)$ is a ring isomorphism.

By using Theorems 3 and 6, and an argument involving Frobenius functors, one establishes Theorem 5.

3. The restriction map. In view of the above results, it is of interest to determine the image of the restriction map

$$
\text { res: } a(G, H) \rightarrow a(B, H)
$$

in as general a situation as possible. Our main result in this direction is

TheOREM 7. Let $H$ be a normal p-subgroup of $G$, where either $G$ is a p-group, or where more generally the p-free part of $[G: H]$ is squarefree. Suppose that the underlying field $\Omega$ is algebraically closed and of characteristic $p$. Let $T$ be the additive subgroup of $a(H, H)$ spanned by all selfconjugate $H$-modules. Then res $a(G, H)=T$.

A vital lemma in the proofs of Theorems 6 and 7 is as follows. 
LEMMA. Let $H$ be a normal p-subgroup of $G$, and suppose that $G / H$ is either a p-group or is cyclic. Let $V$ be any self-conjugate absolutely indecomposable $H$-module. Then there exists a G-module $M$ such that

$$
(M)_{H} \cong V \oplus \Sigma^{\oplus} V_{i}
$$

where each $V_{i}$ is an $H$-module with $\operatorname{dim} V_{i}<\operatorname{dim} V$.

\section{REFERENCES}

1. T. Y. Lam and I. Reiner, Relative Grothendieck groups, J. Algebra 11 (1969), 213-242.

2. - Reduction theorems for relative Grothendieck rings, Trans. Amer. Math. Soc. (to appear).

University of California, Berkeley, California 94720 and

UNIVERSITY OF ILLINOIS, URBANA, ILliNOIS 61801 